TRANSACTIONS OF THE

AMERICAN MATHEMATICAL SOCIETY

Volume 350, Number 6, June 1998, Pages 2265-2276

S $0002-9947(98) 01731-0$

\title{
PERIODIC ORBITS OF THE RESTRICTED THREE-BODY PROBLEM
}

\author{
SALEM MATHLOUTHI
}

\begin{abstract}
We prove, using a variational formulation, the existence of an infinity of periodic solutions of the restricted three-body problem. When the problem has some additional symmetry (in particular, in the autonomous case), we prove the existence of at least two periodic solutions of minimal period $T$, for every $T>0$. We also study the bifurcation problem in a neighborhood of each closed orbit of the autonomous restricted three-body problem.
\end{abstract}

\section{IntroduCtion}

We study the existence of periodic solutions of the restricted three-body problem. We are interested in the configuration studied by Sitnikov [14] and Moser [11]: we consider two mass points of equal mass $m_{1}=m_{2}>0$ moving in the plane under Newton's law of attraction in the elliptic orbits such that the center of mass is at rest. We consider a third mass point moving on the line perpendicular to the plane containing the first two mass points and going through the center of mass, and we suppose that the third mass point does not influence the motion of the first two. Let $z$ be the coordinate describing the motion of the third mass point, so that $z=0$ corresponds to the center of mass of the first two mass points. The restricted three-body problem consists in determining $z$ such that:

$$
-\ddot{z}(t)=\frac{z(t)}{\left(z^{2}(t)+r^{2}(t)\right)^{3 / 2}}
$$

where $r(t)=r(t+2 \pi)$ is the distance from the center of mass to one of the first two mass points. For a small $\varepsilon>0$, the function $r$ takes the form (see Moser [11]):

$$
r(t)=\frac{1}{2}(1-\varepsilon \cos (t))+O\left(\varepsilon^{2}\right) .
$$

In this paper, we suppose that the function $r: R \rightarrow R$ is continuous and $T$-periodic, $T>0$. We prove, using a variational formulation, the existence of an infinity of periodic solutions of the restricted three-body problem. When the $T$-periodic function $r$ satisfies the following hypothesis

$$
\forall t \in[0, T / 2], \quad r^{2}(t)=r^{2}(T-t)
$$

(in particular, in the autonomous case) we prove that for all $k \in N$ the restricted three-body problem has at least two periodic solutions of minimal period $k T$. Finally, for $|\varepsilon| \neq 0$ small enough, we can see the restricted three-body problem as

Received by the editors July 20, 1995.

1991 Mathematics Subject Classification. Primary 34A34; Secondary 34A47.

(C)1998 American Mathematical Society 
a perturbed system of the autonomous system $(\varepsilon=0)$. We prove that in the neighborhood of each orbit:

$$
Z_{k}=\left\{\theta * x_{k}=x_{k}(.+\theta): \theta \in[0, k T]\right\}
$$

where $x_{k}$ is a periodic solution of minimal period $(k T)$ of the autonomous restricted three-body problem, there are at least two $(k T)$-periodic solutions of the perturbed restricted three-body problem for a $|\varepsilon| \neq 0$ small enough.

The existence of an infinity of periodic solutions of the restricted three-body problem was proved for the first time by Moser [11]. The method used by Moser is different from ours. He uses the Bernouilli shift and the symbolic dynamics.

\section{VARIATIONAL FORMULATION}

We consider the restricted three-body problem:

$$
\left\{\begin{array}{l}
-\ddot{z}(t)=\frac{z(t)}{\left(z^{2}(t)+r^{2}(t)\right)^{3 / 2}}, \\
z(0)-z(T)=\dot{z}(0)-\dot{z}(T)=0,
\end{array}\right.
$$

where $T>0$ and $r: R \rightarrow R$ is a continuous $T$-periodic function:

$$
r(t+T)=r(t), \quad \forall t \in R .
$$

We denote by $H_{T}^{1}$ the Hilbert space

$$
H_{T}^{1}=\left\{z \in H^{1}([0, T], R) \mid z(0)=z(T)\right\}
$$

equipped with its usual norm

$$
\|z\|^{2}=\int_{0}^{T}\left(|\dot{z}(t)|^{2}+|z(t)|^{2}\right) d t
$$

and we define the functional $f$ by

$$
\begin{aligned}
H_{T}^{1} & \rightarrow R \\
f: z & \mapsto \int_{0}^{T}\left(\frac{1}{2}|\dot{z}(t)|^{2}+\frac{1}{\sqrt{z^{2}(t)+r^{2}(t)}}\right) d t .
\end{aligned}
$$

It is not difficult to prove that $f$ is of class $C^{2}$ and that the solutions of the system (1) are the critical points of the functional $f$.

We denote by $\mathcal{Z}(f)$ the set of the critical points of $f$ :

$$
\mathcal{Z}(f)=\left\{z \in H_{T}^{1} \mid f^{\prime}(z)=0\right\} .
$$

The Morse index of $z \in \mathcal{Z}(f)$ is defined to be the supremum of the dimensions of the subspaces of $H_{T}^{1}$ on which $f^{\prime \prime}(z)$ is negative definite. The nullity of $z \in \mathcal{Z}(f)$ is defined as the dimension of $\operatorname{ker} f^{\prime \prime}(z)$. We denote by $i(z)$ and $\nu(z)$ respectively, the Morse index and the nullity of the critical point $z$.

Let

$$
m_{0}=\left(\max _{t \in[0, T]}|r(t)|\right)^{3 / 2}
$$

and

$$
k_{1}=\max \left\{k \in N ; k<\frac{T}{2 \pi m_{0}}\right\} .
$$


Theorem 1. If $k_{1} \geq 1$, then

(a) The functional $f$ has at least $4 k_{1}$ of nontrivial critical points, and if $x_{k}$ is a critical point then $-x_{k}$ is also a critical point.

(b) For all $k, 1 \leq k \leq 2 k_{1}$, there exist two critical points $x_{k}, y_{k}$ of $f$ such that

$$
i\left(x_{k}\right) \leq k
$$

and

$$
i\left(y_{k}\right)+\nu\left(y_{k}\right) \geq k
$$

Corollary 1. There exist $p_{0} \in N$ and an increasing sequence of integers $\left(k_{p}\right)_{p=1}^{\infty}$ such that

$$
\lim _{p \rightarrow \infty} k_{p}=+\infty
$$

and, for all $p \geq p_{0}$, the restricted three-body problem has at least $4 k_{p}$ nontrivial $(p T)$-periodic solutions.

Proof of Corollary 1. The function $r$ is $T$-periodic. Then, for all $p \in N, p \geq 1$, it is also a $(p T)$-periodic function. Let

$$
k_{p}=\max \left\{k \in N ; k<\frac{p T}{2 \pi m_{0}}\right\} .
$$

It is clear that $\left(k_{p}\right)_{p=1, \infty}$ is an increasing sequence, that there exists $p_{0} \in N$ such that $k_{p} \geq 1$ for $p \geq p_{0}$ and $\lim _{p \rightarrow \infty} k_{p}=+\infty$. Now, apply Theorem 1 with the period $(p T)$.

Corollary 2. The restricted three-body problem has an infinity of distinct closed orbits.

Proof of Theorem 1. We say that $f$ satisfies the Palais-Smale condition, shortly (P.S.) condition, at the level $c \in R$, if, for every sequence $\left(z_{k}\right) \subset H_{T}^{1}$ such that

$$
f\left(z_{k}\right) \rightarrow c \text { and } f^{\prime}\left(z_{k}\right) \rightarrow 0, \quad \text { as } k \rightarrow \infty
$$

there is a convergent subsequence.

Lemma 1. (a) The functional $f$ is even and satisfies the (P.S.) condition at every level $c>0$.

(b) $Z(f)$ is a compact set.

Proof. (a) It is clear that $f$ is even. Let $\left(z_{k}\right) \subset H_{T}^{1}$ be a sequence such that

$$
f^{\prime}\left(z_{k}\right) h \rightarrow 0, \quad \forall h \in H_{T}^{1}, \text { as } k \rightarrow \infty
$$

and

$$
f\left(z_{k}\right) \rightarrow c>0, \quad \text { as } k \rightarrow \infty .
$$

We denote

$$
E_{0}=\left\{x \in H_{T}^{1} \mid \int_{0}^{T} x(s) d s=0\right\} .
$$

It is not difficult to see that $H_{T}^{1}=E_{0} \oplus R$. Let $\left(x_{k}\right) \subset E_{0}$ and $\left(\xi_{k}\right) \subset R$ be such that

$$
z_{k}=x_{k}+\xi_{k}, \quad \forall k \in N
$$


Let us prove that $\left(z_{k}\right)$ is bounded in $H_{T}^{1}$. Using $(2.2)$, we can see that $\left(\dot{z}_{k}\right)=\left(\dot{x}_{k}\right)$ is bounded in $L^{2}([0, T])$. Then the sequence $\left(x_{k}\right)$ is bounded in $H_{T}^{1}$ and $L^{\infty}([0, T])$. Hence, $\left(z_{k}\right)$ is bounded in $H_{T}^{1}$ if and only if $\left(\xi_{k}\right)$ is bounded in $R$. Suppose that $\left(\xi_{k}\right)$ is not bounded in $R$. Then, up to a subsequence, we can suppose that:

$$
\xi_{k} \rightarrow+\infty, \quad \text { as } k \rightarrow \infty .
$$

The sequence $\left(x_{k}\right)$ is bounded in $H_{T}^{1}$; then

$$
f^{\prime}\left(z_{k}\right) x_{k}=\int_{0}^{T}\left|\dot{z}_{k}\right|^{2}-\frac{\left(x_{k}+\xi_{k}\right) x_{k}}{\left(\left(x_{k}+\xi_{k}\right)^{2}+r^{2}\right)^{3 / 2}} \rightarrow 0 \quad \text { as } k \rightarrow \infty .
$$

On the other hand,

$$
\int_{0}^{T} \frac{\left(x_{k}+\xi_{k}\right) x_{k}}{\left(\left(x_{k}+\xi_{k}\right)^{2}+r^{2}\right)^{3 / 2}} \rightarrow 0 \quad \text { as } k \rightarrow \infty
$$

because $\left(x_{k}\right)$ is bounded in $L^{\infty}$ and $\xi_{k} \rightarrow+\infty$. Then

$$
\int_{0}^{T}\left|\dot{z}_{k}\right|^{2} \rightarrow 0, \quad \text { as } k \rightarrow \infty
$$

For the same reason,

$$
\int_{0}^{T} \frac{1}{\sqrt{\left(x_{k}+\xi_{k}\right)^{2}+r^{2}}} \rightarrow 0, \quad \text { as } k \rightarrow \infty
$$

This implies that

$$
f\left(z_{k}\right) \rightarrow 0, \quad \text { as } k \rightarrow \infty
$$

which is a contradiction with $c>0$.

Thus, $\left(z_{k}\right)$ is bounded in $H_{T}^{1}$. Then, up to subsequence, $\left(z_{k}\right)$ converges weakly in $H_{T}^{1}$ and strongly in $L^{\infty}([0, T])$ to $z \in H_{T}^{1}$. We have

$$
\lim _{k \rightarrow \infty} f^{\prime}\left(z_{k}\right) z=0=f^{\prime}(z) z
$$

and

$$
\lim _{k \rightarrow \infty} f^{\prime}\left(z_{k}\right) z_{k}=\lim _{k \rightarrow \infty} \int_{0}^{T}\left|\dot{z}_{k}\right|^{2}-\frac{z_{k}^{2}}{\left(z_{k}^{2}+r^{2}\right)^{3 / 2}}=0
$$

Then

$$
\lim _{k \rightarrow \infty} \int_{0}^{T}\left|\dot{z}_{k}\right|^{2}=\int_{0}^{T}|\dot{z}|^{2}
$$

and

$$
\lim _{k \rightarrow \infty} \int_{0}^{T} \dot{z}_{k} \dot{z}=\int_{0}^{T}|\dot{z}|^{2}
$$

Hence, $\left(\dot{z}_{k}\right)$ converges to $\dot{z}$ in $L^{2}([0, T])$. Then $\left(z_{k}\right)$ converge to $z$ in $H_{T}^{1}$.

(b) Let $\left(z_{k}\right)$ be a sequence of $\mathcal{Z}(f)$. Let $\left(\xi_{k}\right) \in R,\left(x_{k}\right) \in E_{0}$ be such that

$$
\forall k \in N, \quad z_{k}=x_{k}+\xi_{k} .
$$

It is not difficult to prove that $\left(\dot{z}_{k}\right)=\left(\dot{x}_{k}\right)$ is bounded in $L^{2}([0, T], R)$ and $\left(f\left(z_{k}\right)\right)$ is bounded in $R$. Then, up to a subsequence, we can suppose that $\left(f\left(z_{k}\right)\right)$ converges to $c$ in $R$. If $c>0$, by the (P.S.) condition at the level $c,\left(z_{k}\right)$ has a convergent subsequence. Let us prove that $c>0$. Arguing by contradiction, we suppose that $c=0$. Then, $\left(x_{k}\right)$ converges strongly in $H_{T}^{1}$ and in $L^{\infty}$ to 0 and $\left(\left|\xi_{k}\right|\right)$ goes to $+\infty$ 
as $k \rightarrow \infty$. On the other hand, for all $k \in N, z_{k}$ is a $T$-periodic solution of the restricted three-body problem; hence

$$
0=\int_{0}^{T} \frac{\left(x_{k}(t)+\xi_{k}\right)}{\left(\left(x_{k}+\xi_{k}\right)^{2}+r^{2}(t)\right)^{3 / 2}} d t, \quad \forall k \in N .
$$

This implies that

$$
0=\left|\xi_{k}\right| \xi_{k} \int_{0}^{T} \frac{\left(x_{k}(t)+\xi_{k}\right)}{\left(\left(x_{k}+\xi_{k}\right)^{2}+r^{2}(t)\right)^{3 / 2}} d t, \quad \forall k \in N
$$

which is a contradiction with $T>0$, because, when $k$ goes to $\infty$ the term on the right-hand side goes to $T$.

Let $\Sigma$ denote the family of sets $A \subset H_{T}^{1}-\{0\}$ such that $A$ is closed in $H_{T}^{1}$ and symmetric with respect to 0 . For $A \in \Sigma$, we define the cogenus of $A$ to be

$$
\gamma^{-}(A)=\inf \left\{n \in N \mid \exists \phi: A \rightarrow S^{n-1} \text { odd and continuous }\right\}
$$

and the genus to be

$$
\gamma^{+}(A)=\sup \left\{n \in N \mid \exists \phi: S^{n-1} \rightarrow A \text { odd and continuous }\right\} .
$$

When there does not exist such a $\phi$, we set $\gamma^{ \pm}(A)=\infty$.

The proof of the following properties can be found in [12], [5], [6]:

Proposition 1. $1^{\circ}$. If $x \neq 0, \gamma^{ \pm}(\{x\} \cup\{-x\})=1$.

$2^{\circ}$. If there is an odd map $f \in C(A, B)$, then $\gamma^{ \pm}(A) \leq \gamma^{ \pm}(B)$.

$3^{\circ} . \forall A \in \Sigma, \gamma^{+}(A) \leq \gamma^{-}(A)$.

$4^{\circ}$. If $X$ is a subspace of $H_{T}^{1}$ of dimension $k$ and $S=\{x \in X \mid\|x\|=r\}, r>0$, then $\gamma^{ \pm}(S)=k$.

$5^{\circ}$. If $X$ is a subspace of $H_{T}^{1}$ of codimension $k$ and $A \in \Sigma$ with $\gamma^{-}(A)>k$, then $A \cap X \neq \varnothing$.

For all $k \in N$, we let

$$
\gamma_{k}^{ \pm}=\left\{A \in \Sigma ; A \text { is compact, } \gamma^{ \pm}(A) \geq k\right\}
$$

and

$$
c_{k}^{ \pm}=\inf _{A \in \gamma_{k+1}^{ \pm}} \max _{x \in A} f(x) .
$$

By property 3 of Proposition 1 , for all $k \in N$, we have

$$
c_{k}^{-} \leq c_{k}^{+}
$$

and it is clear that

$$
\gamma_{1}^{ \pm} \supset \gamma_{2}^{ \pm} \supset \cdots \supset \gamma_{k}^{ \pm} \supset \cdots
$$

so we also have

$$
c_{0}^{ \pm} \leq c_{1}^{ \pm} \leq c_{2}^{ \pm} \leq \cdots \leq c_{k}^{ \pm} \leq \cdots
$$

On the other hand, if $f$ satisfies the Palais-Smale condition at the level $c=c_{k}^{ \pm}$, it is known that $c=c_{k}^{ \pm}$is a critical value of $f$; if $c=c_{k}^{-}=c_{k+1}^{-}<f(0)$ and $f$ satisfies the Palais-Smale condition at the level $c$, then $f^{-1}(\{c\})$ contains infinitely many distinct critical points (see [2], [7] and [13]). Hence, to prove Theorem 1, we must prove the existence of $c_{k}^{ \pm}$such that $0<c_{k}^{ \pm}<f(0)$, since $f$ satisfies the Palais-Smale condition at every level $c>0$. 
Proposition 2. (1) $0=c_{0}^{ \pm}<c_{1}^{ \pm}$.

(2) $c_{2 k_{1}}^{ \pm}<f(0)$.

Proof. (1) Let us prove that $c_{0}^{ \pm}=0$. For all $x \in H_{T}^{1}, x \neq 0$, we have $\gamma^{ \pm}(\{x,-x\})=$ 1. Then $c_{0}^{ \pm}=\inf \left\{f(x) ; x \in H_{T}^{1}\right\}$ because

$$
f(x)=\max _{t \in\{x,-x\}} f(t), \quad \forall x \in H_{T}^{1} .
$$

On the other hand, we remark that $\inf \left\{f(x) ; x \in H_{T}^{1}\right\}=0$ because $f$ is a positive functional and $f(\xi) \rightarrow 0$ as $|\xi| \rightarrow+\infty$, if $\xi \in R$.

Let us prove that $c_{1}^{-}>0$. We denote

$$
\delta=\inf _{x \in E_{0}} f(x) .
$$

Using property 5 of Proposition 1, for all $A \in \gamma_{2}^{-}$, we have $A \cap E_{0} \neq \varnothing$, so that

$$
c_{1}^{-} \geq \delta \text {. }
$$

Let $\left(x_{k}\right)$ be a minimizing sequence of $f$ over $E_{0}: f\left(x_{k}\right) \rightarrow \delta$ when $k \rightarrow \infty$. If $\delta=0$, then $\left(x_{k}\right)$ converges to zero in $H_{T}^{1}$ and in $L^{\infty}([0, T])$ so that

$$
\lim _{k \rightarrow \infty} f\left(x_{k}\right)=\lim _{k \rightarrow \infty} \int_{0}^{T}\left(\frac{1}{2}\left|\dot{x}_{k}(t)\right|^{2}+\frac{1}{\sqrt{x_{k}(t)^{2}+r(t)^{2}}}\right) d t=\int_{0}^{T} \frac{1}{|r(t)|} d t>0
$$

which is a contradiction with $\delta=0$. Thus, $\delta>0$ and $c_{1}^{+} \geq c_{1}^{-}>0$.

(2) It is clear that 0 is a critical point of $f$. Let $X$ be the subspace of $H_{T}^{1}$ generated by the following functions:

$1, \sin \left(\frac{2 \pi}{T} t\right), \cos \left(\frac{2 \pi}{T} t\right), \sin \left(\frac{4 \pi}{T} t\right), \cos \left(\frac{4 \pi}{T} t\right), \ldots, \sin \left(\frac{2 k_{1} \pi}{T} t\right), \cos \left(\frac{2 k_{1} \pi}{T} t\right)$.

It is clear that the dimension of $X$ is equal to $2 k_{1}+1$ and that there exists $\alpha>0$ such that

$$
\max _{x \in S} f(x)<f(0)
$$

where $S=\{x \in X \mid\|x\|=\alpha\}$ because $f^{\prime \prime}(0) v . v<0$ for all $v \in X$. Using Proposition 1 , we have $\gamma^{ \pm}(S)=2 k_{1}+1$ and by definition of the $c_{k}^{ \pm}$'s:

$$
c_{2 k_{1}}^{ \pm} \leq \max _{x \in S} f(x)<f(0) .
$$

The proof of (a) of Theorem 1 follows from Proposition 2. To prove (b) of Theorem 1, we need the following proposition (see [3], [4], Proposition 6.8, page $170,[9])$ :

Proposition 3. Let $\Omega$ be a $C^{2}$ open subset of a Hilbert space $H$ and let $f \in$ $C^{2}(\Omega, R)$. Assume $f^{\prime}$ is a Fredholm operator (of null index) on the critical set $\mathcal{Z}(f)=\left\{u \in \Omega, f^{\prime}(u)=0\right\}$. Suppose furthermore that $\mathcal{Z}(f)$ is compact. Then, for any $\varepsilon>0$ and $\eta>0$, there exists $g_{\varepsilon} \in C^{2}(\Omega, R)$ satisfying the following properties:

(g.1) $g_{\varepsilon}(u)=f(u)$, if $\|u-\mathcal{Z}(f)\| \geq \eta$.

(g.2) $\left|g_{\varepsilon}(u)-f(u)\right| \leq \varepsilon,\left\|g_{\varepsilon}^{\prime}(u)-f^{\prime}(u)\right\| \leq \varepsilon, \forall u \in \Omega$.

(g.3) $\left\|g_{\varepsilon}^{\prime \prime}(u)-f^{\prime \prime}(u)\right\|_{\mathcal{L}\left(H, H^{\prime}\right)} \leq \varepsilon, \forall u \in \Omega$.

(g.4) The critical points of $g_{\varepsilon}$ (if any) are in finite number and are nondegenerate.

(g.5) $g_{\varepsilon}^{\prime}$ is a Fredholm operator (of null index) and if $f$ satisfies the (P.S.) condition, then $g_{\varepsilon}$ can also be chosen so that it satisfies the (P.S.) condition. 
It is not difficult to prove that the functional $f$ corresponding to the restricted three-body problem satisfies the assumptions of Proposition 3. Hence, we construct a sequence of functionals $\left(g_{n}\right)$ such that $g_{n}$ satisfies properties (g.1)-(g.5) of Proposition 3 with $\varepsilon=\eta=\frac{1}{n}$ and we can suppose that $g_{n}$ is even for all $n$ (if not, we replace $g_{n}($.$\left.) by \left(g_{n}()+.g_{n}(-).\right) / 2\right)$.

For all $n \in N$, we set

$$
\left(c_{n}\right)_{k}^{ \pm}=\inf _{A \in \gamma_{k+1}^{ \pm}} \max _{x \in A} g_{n}(x)
$$

by (g.2) of Proposition 3 ; it is clear that

$$
c_{k}^{ \pm}-1 / n \leq\left(c_{n}\right)_{k}^{ \pm} \leq c_{k}^{ \pm}+1 / n, \quad \forall k \in N .
$$

Then, for $n$ large enough, $\left(c_{n}\right)_{k}^{ \pm}\left(k=1,2, \ldots, 2 k_{1}\right)$ are critical values of $g_{n}$. In [5] (see also [6]) Bahri and Lions proved the existence of at least two critical points of $g_{n}, x_{k}^{n}$ and $y_{k}^{n}$, for $k=1,2, \ldots, 2 k_{1}$, critical points of $g_{n}$ such that

$$
\left(c_{n}\right)_{k}^{+}=g_{n}\left(y_{k}^{n}\right), \quad i_{n}\left(y_{k}^{n}\right)+\nu_{n}\left(y_{k}^{n}\right) \geq k,
$$

and

$$
\left(c_{n}\right)_{k}^{-}=g_{n}\left(x_{k}^{n}\right), \quad i_{n}\left(x_{k}^{n}\right) \leq k,
$$

where $i_{n}$ and $\nu_{n}$ are, respectively, the Morse index and the nullity of $x_{k}^{n}$ and $y_{k}^{n}$, as critical points of the functional $g_{n}$. Now, using Proposition 3 and the Palais-Smale condition, we can prove (b) of Theorem 1.

Remark 1. (a) It is not difficult to see that, for any $T$-periodic solution $x$ of the restricted three-body problem, the dimension of the space of solutions of the linearized system

$$
\left\{\begin{array}{l}
\ddot{y}(t)=\frac{2 x^{2}(t)-r^{2}(t)}{\left(x^{2}(t)+r^{2}(t)\right)^{5 / 2}} y(t), \\
y(0)-y(T)=\dot{y}(0)-\dot{y}(T)=0
\end{array}\right.
$$

is equal to the nullity of the critical point $x$ of $f$. Then the nullity of any critical point $x$ of $f$ satisfies $0 \leq \nu(x) \leq 2$.

(b) In the autonomous case, $\left(r()=.r_{0}\right)$, if $x$ is a $T$-periodic solution of the restricted three-body problem, then $y=\dot{x}$ is a solution of the linearized problem (2). Thus, $\nu(x) \geq 1$. Using a theorem due to Willem [15] (see also [10], page 228), we can prove that the nullity of any critical point $x$ of $f$ is equal to one.

\section{VARIATIONAL FORMULATION With A SYMMETRY CONDITION ON $r$}

In this section, we assume that the $T$-periodic function $r$ satisfies the following symmetry condition (see [8] for another application):

$$
\forall t \in[0, T / 2], \quad r^{2}(t)=r^{2}(T-t) .
$$

We consider the system

$$
\left\{\begin{array}{l}
-\ddot{u}(t)=\frac{u(t)}{\left(u^{2}(t)+r^{2}(t)\right)^{3 / 2}}, \quad t \in[0, p T / 2], \\
u(0)=u(p T / 2)=0 .
\end{array}\right.
$$

We denote by $E_{p}, p \in N^{*}$, the Hilbert space

$$
E_{p}=\left\{u \in H^{1}([0, p T / 2], R) \mid u(0)=u(p T / 2)=0\right\}
$$


equipped with its usual norm

$$
\|u\|^{2}=\int_{0}^{p T / 2}|\dot{u}(t)|^{2} d t
$$

and we define the functional $f_{p}$ by

$$
\begin{aligned}
& E_{p} \rightarrow R \\
& f_{p}: u \mapsto \int_{0}^{p T / 2}\left(\frac{1}{2}|\dot{u}(t)|^{2}+\frac{1}{\sqrt{u^{2}(t)+r^{2}(t)}}\right) d t .
\end{aligned}
$$

It is not difficult to prove that $f_{p}$ is of class $C^{1}$ and that the solutions of system $(3)_{p}$ are the critical points of the functional $f_{p}$.

Theorem 2. For all $p \in N$, let $m_{0}$ and $k_{p}$ be such that

$$
m_{0}=\left(\max _{t \in[0, T / 2]}|r(t)|\right)^{3 / 2}, \quad k_{p}=\max \left\{k \in N ; k<\frac{p T}{2 \pi m_{0}}\right\} .
$$

If $k_{p} \geq 1$, the functional $f_{p}$ has at least two critical points $u_{p},-u_{p}$ such that

$$
\left.u_{p}(t) \neq 0, \quad \forall t \in\right] 0, p T / 2[.
$$

Corollary 3. There exists $p_{0} \in N$ such that, for all $p \in N, p \geq p_{0}$, the restricted three-body problem has at least two nontrivial periodic solutions, $x_{p},-x_{p}$, of minimal period $(p T)$ such that:

$$
x_{p}(t+p T / 2)=-x_{p}(p T / 2-t), \quad \forall t \in[0, p T / 2] .
$$

Proof of Corollary 3. The sequence $\left(k_{p}\right)$ of Theorem 2 is an increasing sequence such that

$$
\lim _{p \rightarrow \infty} k_{p}=+\infty
$$

Then, there exists $p_{0}$ such that, for all $p \in N, p \geq p_{0}, k_{p} \geq 1$. Starting from the critical point $u_{p}$ of $f_{p}$, we construct a $(p T)$-periodic solution of the restricted three-body problem by putting

$$
x_{p}(t)= \begin{cases}u_{p}(t), & t \in[0, p T / 2], \\ -u_{p}(p T-t), & t \in[p T / 2, p T],\end{cases}
$$

and then extending $x_{p}$ by $(p T)$-periodicity, to the whole real line. It is not difficult to see that $x_{p}$ is a $(p T)$-periodic solution of the restricted three-body problem and it is clear that the minimal period of $x_{p}$ is $(p T)$ because $u_{p}$ satisfies (2.3).

Proof of Theorem 2.

Lemma 2. The functional $f_{p}$ is even, bounded from below in $E_{p}$ and satisfies the (P.S.) condition at every level $c \in R$.

Proof of Lemma 2. It is clear that $f_{p}$ is even and bounded from below. Let $\left(z_{k}\right) \subset$ $E_{p}$ be a sequence such that:

$$
f_{p}^{\prime}\left(z_{k}\right) h \rightarrow 0, \quad \forall h \in E_{p}, \quad \text { as } k \rightarrow \infty
$$

and

$$
f_{p}\left(z_{k}\right) \rightarrow c \in R, \quad \text { as } k \rightarrow \infty
$$


Then $\left(z_{k}\right)$ is bounded in $E_{p}$. Up to a subsequence, $\left(z_{k}\right)$ converges weakly in $E_{p}$ and strongly in $L^{\infty}([0, p T / 2])$ to $z \in E_{p}$. We have

$$
\lim _{k \rightarrow \infty} f_{p}^{\prime}\left(z_{k}\right) z=0=f_{p}^{\prime}(z) z
$$

and

$$
\lim _{k \rightarrow \infty} f_{p}^{\prime}\left(z_{k}\right) z_{k}=\lim _{k \rightarrow \infty} \int_{0}^{p T / 2}\left|\dot{z}_{k}\right|^{2}-\frac{z_{k}^{2}}{\left(z_{k}^{2}+r^{2}\right)^{3 / 2}}=0
$$

Then

$$
\lim _{k \rightarrow \infty} \int_{0}^{p T / 2}\left|\dot{z}_{k}\right|^{2}=\int_{0}^{p T / 2}|\dot{z}|^{2}
$$

and

$$
\lim _{k \rightarrow \infty} \int_{0}^{p T / 2} \dot{z}_{k} \dot{z}=\int_{0}^{p T / 2}|\dot{z}|^{2}
$$

Hence, $\left(\dot{z}_{k}\right)$ converges to $\dot{z}$ in $L^{2}([0, p T / 2])$. Then $\left(z_{k}\right)$ converges to $z$ in $E_{p}$.

Lemma 3. There exists $p_{0} \in N$ such that, for all $p \geq p_{0}$, the minimum of $f_{p}$ is achieved in $u_{p} \in E_{p}$ such that

$$
0<f_{p}\left(u_{p}\right)=\min \left\{f_{p}(u) ; u \in E_{p}\right\}<f_{p}(0), \quad \forall p \geq p_{0} .
$$

Proof of Lemma 3. With the (P.S.) condition, it is easy to see that the minimum of the functional $f_{p}$ is achieved. For all $p \in N$, let $u_{p}$ be such that

$$
f_{p}\left(u_{p}\right)=\min \left\{f_{p}(u) ; u \in E_{p}\right\} .
$$

If $k_{p} \geq 1$, we define $w_{p} \in E_{p}$ as follows:

$$
w_{p}(t)=\sin \left(\frac{2 \pi}{p T} k_{p} t\right), \quad \forall t \in[0, p T / 2] .
$$

We have

$$
\begin{aligned}
f_{p}^{\prime \prime}(0) w_{p} w_{p} & =\int_{0}^{p T / 2}\left(\dot{w}_{p}(t)\right)^{2} d t-\int_{0}^{p T / 2} \frac{\left(w_{p}(t)\right)^{2}}{r^{3}(t)} d t \\
& \leq\left(\left(\frac{2 \pi}{p T} k_{p}\right)^{2}-\frac{1}{m_{0}^{2}}\right) p T / 4 \\
& <0 .
\end{aligned}
$$

Then

$$
f_{p}\left(u_{p}\right)<f_{p}(0)
$$

and it is not difficult to see that

$$
f_{p}(u)>0, \quad \forall p, \forall u .
$$

By Lemma 2 and Lemma 3, we have the existence of a critical point $u_{p}$ of the functional $f_{p}$. To prove (2.4), we can suppose that

$$
u_{p}(t) \geq 0, \quad t \in\left[0, p \frac{T}{2}\right]
$$

because $\left|u_{p}\right|$ is also in $E_{p}$ and $f_{p}\left(\left|u_{p}\right|\right)=f_{p}\left(u_{p}\right)$. On the other hand, $u_{p}$ is a critical point of $f_{0}$; thus $u_{p}$ satisfies the system $(3)_{p}$. Now, using $(3)_{p}$ and (2.7), we deduce (2.4). 


\section{BifurCATION IN THE RESTRICTED THREE-BODY PROBLEM}

In this section, we are interested in the case $T=2 \pi$ and $r=r_{\varepsilon}, \varepsilon \in R$, is given by Moser [11]:

$$
r_{\varepsilon}(t)=\frac{1}{2}(1-\varepsilon \cos (t))+O\left(\varepsilon^{2}\right)
$$

and we suppose that $r=r_{\varepsilon}$ is $C^{\infty}$ in $\varepsilon$ and $t$.

For all $p \geq 1$ and for $|\varepsilon| \neq 0$ small enough, we can see the restricted three-body problem

$$
\left\{\begin{array}{l}
-\ddot{z}(t)=\frac{z(t)}{\left(z^{2}(t)+r_{\varepsilon}^{2}(t)\right)^{3 / 2}}, \\
z(0)-z(2 p \pi)=\dot{z}(0)-\dot{z}(2 p \pi)=0
\end{array}\right.
$$

as a perturbed system of the autonomous restricted three-body problem for $\varepsilon=0$ :

$$
\left\{\begin{array}{l}
-\ddot{z}(t)=\frac{z(t)}{\left(z^{2}(t)+\frac{1}{4}\right)^{3 / 2}}, \\
z(0)-z(2 p \pi)=\dot{z}(0)-\dot{z}(2 p \pi)=0 .
\end{array}\right.
$$

Proposition 4. For all $p \geq 1$, the autonomous restricted three-body problem $(1)_{0}^{p}$ has at least two nontrivial periodic solutions $x_{p},-x_{p}$ of minimal period $2 p \pi$.

Proof. For all $p \in N, p \geq 1$, we have

$$
\begin{aligned}
m_{0} & =\left(\max _{t \in[0, \pi]}\left|r_{0}(t)\right|\right)^{3 / 2}=\frac{1}{\sqrt{8}}, \\
k_{p} & =\max \{k \in N ; k<p \sqrt{8}\} .
\end{aligned}
$$

Then $k_{p} \geq 1$ for all $p \geq 1$. By Theorem 2 and Corollary 3 , we have Proposition 4 .

Remark 2. The system $(1)_{0}^{p}$ is autonomous. If $x$ is a solution of $(1)_{0}^{p}$, then, for all $\tau \in[0,2 p \pi[, \tau * x=x(.+\tau)$ is also a solution. Hence, we have an orbit of solutions:

$$
Z(x)=\{\tau * x=x(.+\tau): \tau \in[0,2 p \pi]\} .
$$

Theorem 3. There exists $\bar{\varepsilon}>0$ such that: for all $\varepsilon, 0<|\varepsilon|<\bar{\varepsilon}$, the perturbed restricted three-body problem $(1)_{\varepsilon}^{p}$ has at least two $(2 p \pi)$-periodic solutions near the orbit $Z\left(x_{p}\right)$, where $x_{p}$ is the $(2 p \pi)$-periodic solution given by Proposition 4 .

Theorem 3 is a particular case of a general theorem of [1]. Here, we use the implicit function theorem and the following lemmas:

Lemma 4. Let $a: R \rightarrow R, f: R \rightarrow R$ be two T-periodic functions. Let $E_{0}$ be the space of solutions of the linear system:

$$
\ddot{y}(t)=a(t) y(t), \quad y(0)-y(T)=\dot{y}(0)-\dot{y}(T)=0 .
$$

Then the linear system

$$
\ddot{y}(t)=a(t) y(t)+f(t), \quad y(0)-y(T)=\dot{y}(0)-\dot{y}(T)=0
$$

has a solution if and only if $\int_{0}^{T} f(t) v(t) d t=0$ for all $v \in E_{0}$. 
Lemma 4 is a classical result for the problem with periodic boundary conditions. We define the function $F: R \times H_{2 p \pi}^{1} \rightarrow H_{2 p \pi}^{1}$ by

$$
\langle F(\varepsilon, z), h\rangle=\int_{0}^{2 p \pi}\left(\dot{z}(t) \dot{h}(t)-\frac{z(t) h(t)}{\left(z^{2}(t)+r_{\varepsilon}^{2}(t)\right)^{3 / 2}}\right) d t, \quad \forall h \in H_{2 p \pi}^{1}
$$

where $\langle.,$.$\rangle is the scalar product in H_{2 p \pi}^{1}$. It is not difficult to prove that $F$ is $C^{\infty}$ and

$$
F(\varepsilon, z)=0 \Leftrightarrow z \text { is a solution of }(1)_{\varepsilon}^{p} .
$$

We denote by $\mathcal{L}_{\tau}=F_{z}^{\prime}\left(0, \tau * x_{p}\right)$ and $E_{\tau}$ the subspace of $H_{2 p \pi}^{1}$ generated by $y=$ $\tau * \dot{x}_{p}$.

Remark 3. By Remark 2 and Lemma 4, we can deduce that

$$
\operatorname{Ker}\left(\mathcal{L}_{\tau}\right)=E_{\tau} \text {, and Range }\left(\mathcal{L}_{\tau}\right)=E_{\tau}^{\perp} .
$$

Lemma 5. There exist $\varepsilon_{0}>0$ and two $C^{\infty}$ functions:

$$
\lambda:]-\varepsilon_{0}, \varepsilon_{0}[\times R \rightarrow R, \quad z:]-\varepsilon_{0}, \varepsilon_{0}\left[\times R \rightarrow H_{2 p \pi}^{1}\right.
$$

such that

(a) $\left.z(0, \tau)=\tau * x_{p}, z(\varepsilon, \tau+2 p \pi)=z(\varepsilon, \tau), \forall \tau \in R, \forall \varepsilon \in\right]-\varepsilon_{0}, \varepsilon_{0}[$,

(b) $\left.F(\varepsilon, z(\varepsilon, \tau))+\lambda(\varepsilon, \tau) \tau * \dot{x}_{p}=0, \forall \tau \in R, \forall \varepsilon \in\right]-\varepsilon_{0}, \varepsilon_{0}[$,

(c) $z\left(\varepsilon, \tau_{1}\right)(.) \neq z\left(\varepsilon, \tau_{2}\right)(),. \forall \tau_{1}, \tau_{2} \in\left[0,2 p \pi\left[, \tau_{1} \neq \tau_{2}, \forall \varepsilon \in\right]-\varepsilon_{0}, \varepsilon_{0}[\right.$.

Proof of Lemma 5. Let $G: R \times R \times R \times H_{2 p \pi}^{1} \rightarrow R \times H_{2 p \pi}^{1}$ be defined by

$$
G(\varepsilon, \tau, \lambda, z)=\left(\left\langle z, \tau * \dot{x}_{p}\right\rangle, F(\varepsilon, z)+\lambda \tau * \dot{x}_{p}\right) .
$$

Using Remark 3, it is not difficult to see that

$$
G\left(0, \tau, 0, \tau * x_{p}\right)=0 \text { and } G_{(\lambda, z)}^{\prime}\left(0, \tau, 0, \tau * x_{p}\right) \text { is an isomorphism }
$$

for all $\tau \in[0, p T]$. Then we can apply the implicit function theorem for the function $G$ in the point $\left(0, \mu, 0, \mu * x_{p}\right)$ for all $\mu \in[0,2 p \pi]$ : there exist $\varepsilon_{\mu}>0$, an open neighborhood $V(\mu)$ of $\mu$ and a unique pair of $C^{\infty}$ functions

$$
\left.\lambda_{\mu}:\right]-\varepsilon_{\mu}, \varepsilon_{\mu}\left[\times V(\mu) \rightarrow R, \quad z_{\mu}:\right]-\varepsilon_{\mu}, \varepsilon_{\mu}\left[\times V(\mu) \rightarrow H_{2 p \pi}^{1}\right.
$$

such that

$$
\left.0=G\left(\varepsilon, \tau, \lambda_{\mu}(\varepsilon, \tau), z_{\mu}(\varepsilon, \tau)\right), \quad \forall(\varepsilon, \tau) \in\right]-\varepsilon_{\mu}, \varepsilon_{\mu}[\times V(\mu) .
$$

Now, using the $2 p \pi$ periodicity of $G$ in $\tau$, the unicity of the functions $\lambda_{\mu}, z_{\mu}$ and the compactness of $[0,2 p \pi]$, we can find $\varepsilon_{0}>0$ and construct two functions $\lambda, z$ that satisfy (a) and (b) of Lemma 5. Let $\tau_{1} \neq \tau_{2} \in R$ be such that $0 \leq \tau_{1}<\tau_{2}<2 p \pi$. It is clear that $\tau_{1} * x_{p} \neq \tau_{2} * x_{p}$, because $0<\tau_{2}-\tau_{1}<2 p \pi$ and $2 p \pi$ is the minimal period of $x_{p}$. On the other hand, $z$ is a continuous function of $\varepsilon$ and $\tau$; then we can choose $\varepsilon_{0}>0$ such that (c) is true.

Proof of Theorem 3. Let $\varepsilon$ be such that $0<|\varepsilon|<\varepsilon_{0}$. We define the function $h_{\varepsilon}: R \rightarrow R$ by

$$
h_{\varepsilon}(\tau)=\int_{0}^{2 p \pi}\left(\frac{1}{2}|\dot{z}(t)|^{2}+\frac{1}{\sqrt{z^{2}(t)+r_{\varepsilon}^{2}(t)}}\right) d t
$$


where $z=z(\varepsilon, \tau)$ is the function given by Lemma 5 . Using Lemma 5 , it is not difficult to see that $h_{\varepsilon}$ is a $C^{\infty} 2 p \pi$-periodic function and

$$
h_{\varepsilon}^{\prime}(\tau)=\left\langle F(\varepsilon, z(\varepsilon, \tau)), \frac{\partial z(\varepsilon, \tau)}{\partial \tau}\right\rangle=-\lambda(\varepsilon, \tau)\left\langle\tau * \dot{x}_{p}, \frac{\partial z(\varepsilon, \tau)}{\partial \tau}\right\rangle
$$

and

$$
\left\langle\tau * \dot{x}_{p}, \frac{\partial z(0, \tau)}{\partial \tau}\right\rangle=\left\langle\tau * \dot{x}_{p}, \tau * \dot{x}_{p}\right\rangle \neq 0
$$

then the continuity of $\frac{\partial z}{\partial r}$ in $\varepsilon$ implies that there exists $0<\bar{\varepsilon} \leq \varepsilon_{0}$ such that

$$
\left\langle\tau * \dot{x}_{p}, \frac{\partial z(\varepsilon, \tau)}{\partial \tau}\right\rangle \neq 0, \quad \forall \varepsilon,|\varepsilon|<\bar{\varepsilon}
$$

Hence, for all $\varepsilon \in]-\bar{\varepsilon}, \bar{\varepsilon}\left[\right.$, if $\bar{\tau}$ is a critical point of $h_{\varepsilon}$, then $F(\varepsilon, z(\varepsilon, \bar{\tau}))=0$.

On the other hand, $h_{\varepsilon}$ achieves its maximum and minimum in two points $\tau_{1} \neq$ $\tau_{2} \in\left[0,2 p \pi\right.$ [ because $h_{\varepsilon}$ is continuous and $2 p \pi$-periodic. Then $z\left(\varepsilon, \tau_{i}\right), i=1,2$, are two $2 p \pi$-periodic solutions of $(1)_{\varepsilon}^{p}$ and by the continuity of $z$ in $\varepsilon$, we can see that the two solutions are in a neighborhood of the orbit $Z\left(x_{p}\right)$.

\section{REFERENCES}

1. A. Ambrosetti, V. Coti Zelati and I. Ekeland, Symmetry breaking in Hamiltonian systems, J. Differential Equations 67 (1987), 165-184. MR 88h:58040

2. A. Ambrosetti and P. H. Rabinowitz, Dual variational methods in critical point theory and applications, J. Funct. Anal. 14 (1973), 349-381. MR 51:6412

3. A. Bahri, Une méthode perturbative en théorie de Morse, Thèse d'Etat, Publications de l'Université Paris VI, 1981.

4. A. Bahri and H. Berestycki, A perturbation method in critical point theory and application, Trans. Amer. Math. Soc. 267 (1981), 1-32.

5. A. Bahri and P. L. Lions, Morse index of some min-max critical points, I. Applications to multiplicity results, Comm. Pure Appl. Math. 41 (1988), 1027-1037. MR 90b:58035

6. K. C. Chang, Infinite Dimensional Morse Theory and Multiple Solution Problems, Progress in Nonlinear Differential Eqs. and their Appl., Vol. 6, Birkhäuser, 1993. MR 87m:58032

7. D. C. Clark, A variant of the Ljusternik-Schnirelman theory, Indiana Univ. Math. J. 22 (1972/73), 65-74. MR 45:5836

8. M. Girardi and M. Matzeu, Periodic solutions of second order nonautonomous systems with the potentials changing sign, Rend. Math. Acc. Lincei. 4 (1993), 273-277. MR 95c:58038

9. A. Marino and G. Prodi, Metodi perturbativi nella teoria di Morse, Boll. Un. Math. Ital. 11 (1975), 1-32. MR 54:6192

10. J. Mawhin and M. Willem, Critical point theory and Hamiltonian systems, Springer, 1989. MR 90e: 58016

11. J. Moser, Stable and random motions in dynamical systems, Ann. Math. Studies 77, Princeton Univ. Press, 1973. MR 56:1355

12. P. H. Rabinowitz, Minimax Methods in Critical Point Theory with Applications to Differential Equations, CBMS Reg. Conf. Ser. in Math. No. 65, Amer. Math. Soc., Providence, R.I., 1986. MR 87k:58024

13. W Variational methods for nonlinear eigenvalue problems, Eigenvalues of Nonlinear Problems, Edizioni Cremonese, Roma, 1974. MR 57:4232

14. K. Sitnikov, Existence of oscillating motion for the three-body problem, J. Dokl. Akad. Nauk USSR 133 (1960), 303-306.

15. M. Willem, Perturbations of nondegenerate periodic orbits of Hamiltonian systems, Periodic Solutions of Hamiltonian Systems and Related Topics, Rabinowitz, Ambrosetti, Ekeland, Zehnder, eds., Reidel, Dordrecht, 1987, pp. 261-266. MR 89c:58047

Faculté des Sciences de Tunis, Département de Mathématiques, Campus Universitaire, 1060, Tunis, Tunisie 